\title{
Legislación sobre aprovechamiento de recursos naturales en Latinoamérica
}

Legislation about utilization natural resources in Latin America

\author{
Roa Vega María Ligia ${ }^{1}$ \\ 'Zootecnista MSc. Docente Escuela de Ciencias Animales, Universidad de los \\ Llanos \\ mroa@unillanos.edu.co
}

Recibido 05 de Marzo 2013, Aceptado 15 de julio 2013

\section{RESUMEN}

Durante la última década ha aumentado significativamente la atención de las organizaciones públicas y privadas en lo referente al medio ambiente, debido a las implicaciones que el deterioro y agotamiento de los recursos han ocasionado sobre las poblaciones y los sectores productivos. En esta medida, los gobiernos han generado una serie de condiciones para el uso o aprovechamiento de los recursos naturales, reglamentando estas disposiciones por medio de leyes, resoluciones o decretos. Se considera el medio ambiente como patrimonio común y que es obligación de los gobiernos y personas protegerlo, y de velar por su conservación. También se consideran los bienes de uso público, los parques naturales, las tierras comunales de grupos étnicos, las tierras de resguardo, el patrimonio arqueológico y que, son inalienables, imprescriptibles e inembargables. Por lo tanto se proponen acciones de desarrollo sostenible para lograr el crecimiento económico, la elevación de la calidad de vida y el bienestar social, sin agotar la base de los recursos naturales renovables en que se sustenta, ni deteriorar el medio ambiente o el derecho de las generaciones futuras a utilizarlo para la satisfacción de sus propias necesidades, por lo tanto es deber del estado planificar el manejo y aprovechamiento de los recursos naturales para garantizar su desarrollo sostenible, su conservación o sustitución. Para dar cumplimiento a las disposiciones es necesario realizar un Estudio de Impacto Ambiental (EIA), que se debe presentar ante la autoridad ambiental competente, el peticionario contendrá información sobre la localización del proyecto, y los elementos 
abióticos, bióticos y socioeconómicos del medio, que puedan sufrir deterioro por la respectiva obra o actividad, para cuya ejecución se pide licencia, y la evaluación de los impactos. Además debe incluir el diseño de los planes de prevención, corrección y compensación de impactos y el plan de manejo ambiental de la obra o actividad. Una vez tomada la decisión de realizar el proyecto se pasa a la fase de recogida de información acerca del proyecto y del medio afectado: En ese orden de ideas, las organizaciones se ven en la obligación de planear diseñar e implementar el sistema de gestión ambiental, concebida como aquel que identifica aspectos e impactos significativos al mismo tiempo que propone, implementa y revisa acciones dirigidas a la prevención, corrección o mitigación de estos impactos.

Palabras clave: Leyes gubernamentales, conservación, medio ambiente.

\begin{abstract}
During the past decade has significantly increased the attention of public and private organizations in relation to the environment, due to the implications that the deterioration and resource depletion have caused on stocks and sectors. To this extent, governments have generated a series of conditions for the use or exploitation of natural resources, regulating these provisions by laws, orders or decrees. It considers the environment as a common heritage and it is the duty of governments and individuals protect, and ensure the protection of the environment. Also considered are public goods, natural parks, communal lands of ethnic groups, land slip, and archaeological heritage, are inalienable and indefeasible. Therefore proposed Sustainable development actions to achieve economic growth, raising the quality of life and social welfare, without depleting the natural resource base that underpins renewable, or damaging the environment or the right to use future generations to satisfy their needs, therefore it is the duty of the state to plan the management and use of natural resources to ensure sustainable development, preservation or replacement. To comply with the provisions is required Environmental Impact Study (EIS), the set of information that must be submitted to the competent environmental authority the petitioner contain information on the
\end{abstract}


location of the project, and elements abiotic, biotic and socioeconomic environment, which are subject to deterioration by the respective work or activity, the performance of which ask permission, and evaluation of impacts. Also include the design of plans for prevention, correction and compensation of impacts and the environmental management plan of the work or activity. Once the decision to implement the project is passed to the phase of collecting information about the project and the environment affected: In that vein, organizations are obliged to plan design and implement environmental management system, conceived as one that identifies significant aspects and impacts while proposes, implements and reviews actions aimed at the prevention, correction or mitigate these impacts.

Keywords: Government laws, conservation, environment.

\section{INTRODUCCIÓN}

La investigación en gestión ambiental ha surgido en el contexto de la globalización como una dimensión re-orientadora del desarrollo que califica el crecimiento económico al remitirse a los fundamentos mismos de la producción y el consumo. De esta manera, el desarrollo sostenible se apoya en el reconocimiento de la función que cumple el medio ambiente y los recursos naturales como base de sustentación material, ecosistémica, ambiental y energética de los procesos económicos. El desarrollo sostenible debe convertirse en el pilar de la transición de la modernidad hacia una posmodernidad caracterizada por cambios en los modos de producción y consumo acompañados de la innovación tecnológica con fundamentos en la investigación ambiental, para lograr la competitividad económica, por la valoración metodológica de la diversidad natural y cultural, lo cual se basa en fortalecimiento de lo local democrático debido a los avances en la comunicación y los sistemas de información (CEPAL, 2000).

Es claro que a los problemas tradicionales de pobreza y desigualdad, se añaden los límites y requisitos ecológicos así como la necesidad de revertir los procesos de deterioro ambiental para lograr un desarrollo sostenible y equitativo dentro del contexto de globalización económica. En el ámbito nacional es urgente superar la 
degradación de la calidad del agua, del suelo y del aire, especialmente en las zonas urbanas que hoy albergan a más de tres cuartas partes de la población mundial, desertificación y pérdidas de biodiversidad y de suelo para garantizar la sostenibilidad de la producción agropecuaria, minera y forestal. Se han alcanzado umbrales riesgosos en la explotación de los recursos naturales que requieren un cambio de rumbo ya que se han incrementado las actividades productivas exponencialmente frente a una dotación limitada de recursos ecológicos. Lo anterior demuestra la insostenibilidad de los ecosistemas, poniendo en tela de juicio los propios patrones culturales y relaciones entre seres humanos y la naturaleza (CEPAL, 2000).

La investigación en gestión ambiental como dimensión esencial del desarrollo sostenible demanda políticas públicas modernas acompañadas de conocimientos e instrumentos interdisciplinarios, lo que implica un reto sociopolítico de gran envergadura al estar marcada por el conflicto de intereses de múltiples actores económicos. Esto presenta a los gobiernos el importante reto de emplazar nuevos marcos institucionales e instrumentales de gestión ambiental que puedan reorientar el desarrollo futuro hacia patrones de producción y consumo compatibles con la sostenibilidad y a la vez reducir la pobreza en varias regiones del mundo (EPA, 2009).

En el caso de América Latina y el Caribe, se presentan condiciones ecológicas, económicas y sociales que le proveen de innumerables ventajas comparativas en función de los servicios ambientales globales para ser más competitivos en el contexto de la globalización. Su enorme diversidad natural y cultural y los enfoques innovadores de integración regional logrados en Centroamérica son ejemplos relevantes de esta situación privilegiada. Un enorme desafío a vencer es la actual disociación entre las políticas públicas y las preferencias ciudadanas, pues en el debate del desarrollo sostenible, hay que tener en cuenta varios actores no-estatales, reconocidos en la comunidad científica y el sector privado y la sociedad civil organizada. No cabe duda, que actualmente, las cuestiones ambientales, que fundamentan la calidad de vida junto con la noción de 
distribución equitativa de costos y beneficios ambientales y de apropiación de recursos naturales están adquiriendo mayor relevancia en la agenda política y ciudadana. Un paso esencial para lograr la convergencia entre crecimiento económico y sostenibilidad consiste en remplazar un marco de políticas e instituciones basadas en la investigación de gestión ambiental para lograr políticas acertadas que regulen conductas e induzcan los incentivos de sostenibilidad (EPA, 2009 y CEPAL 2000).

\section{MARCO LEGAL}

Analizando algunas normas para el aprovechamiento de los recursos naturales de algunos países latinoamericanos, incluido Colombia, se observa una gran disposiciones cuidadosas al respecto, porque no solamente es una inquietud nacional sino mundial, por ejemplo en Colombia la Constitución Política de Colombia de 1991 (CPC) elevó a norma constitucional la consideración, manejo y conservación de los recursos naturales y el medio ambiente, a través de varios principios fundamentales:

1-Derecho a un ambiente sano: En su Artículo 79, consagra que: "Todas las personas tienen derecho a gozar de un ambiente sano. La Ley garantizará la participación de la comunidad en las decisiones que puedan afectarlo. Es deber del Estado proteger la diversidad e integridad del ambiente, conservar las áreas de especial importancia ecológica y fomentar la educación para el logro de estos fines". Esta norma puede interpretarse de manera solidaria con el principio fundamental del derecho a la vida, ya que éste sólo se podría garantizar bajo condiciones en las cuales la vida pueda disfrutarse con calidad.

2-El medio ambiente como patrimonio común: La (CPC) incorpora este principio al imponer al Estado y a las personas la obligación de proteger las riquezas culturales y naturales (Art. 8), así como el deber de las personas y del ciudadano de proteger los recursos naturales y de velar por la conservación del ambiente (Art. 95). En desarrollo de este principio, en el Art. 58 consagra que: "La propiedad es una función social que implica obligaciones y, como tal, le es 
inherente una función ecológica"; continúa su desarrollo al determinar en el Art. 63 que: "Los bienes de uso público, los parques naturales, las tierras comunales de grupos étnicos, las tierras de resguardo, el patrimonio arqueológico de la Nación y los demás bienes que determine la Ley, son inalienables, imprescriptibles e inembargables" (EPC, 1991).

3-Desarrollo Sostenible: Definido como el desarrollo que conduce al crecimiento económico, a la elevación de la calidad de vida y al bienestar social, sin agotar la base de los recursos naturales renovables en que se sustenta, ni deteriorar el medio ambiente $\mathrm{o}$ el derecho de las generaciones futuras a utilizarlo para la satisfacción de sus propias necesidades, la CPC en desarrollo de este principio, consagró en su Art. $\mathbf{8 0}$ que: "El Estado planificará el manejo y aprovechamiento de los recursos naturales para garantizar su desarrollo sostenible, su conservación o sustitución. Además, deberá prevenir y controlar los factores de deterioro ambiental, imponer las sanciones legales y exigir la reparación de los daños causados. Así mismo, cooperará con otras naciones en la protección de los ecosistemas situados en zonas fronterizas". Lo anterior implica asegurar que la satisfacción de las necesidades actuales se realice de una manera tal que no comprometa la capacidad y el derecho de las futuras generaciones para satisfacer las propias" (EPC, 1991).

Analizando la constitución política del Perú (CPP) en el capítulo Il del ambiente y los recursos naturales: 1-Artículos 66.- "Los recursos naturales, renovables y no renovables, son patrimonio de la nación. El estado es soberano en su aprovechamiento. Por ley orgánica se fijan las condiciones de su utilización y de su otorgamiento a particulares. La concesión otorga a su titular un derecho real, sujeto a dicha norma legal". 2-Artículo 67.- "El Estado determina la política nacional del ambiente. Promueve el uso sostenible de sus recursos naturales. 3Artículo 68.- "El Estado está obligado a promover la conservación de la diversidad biológica y de las áreas naturales protegidas". 4-Artículo 69.- "El Estado promueve el desarrollo sostenible de la Amazonía con una legislación adecuada" (ECCDP, 2009). 
En la República de Argentina existe una ley general del ambiente que se busca que éste bien quede jurídicamente protegido; en su Artículo 1ํe establece los presupuestos mínimos para el logro de una gestión sustentable y adecuada del ambiente, la preservación y protección de la diversidad biológica y la implementación del desarrollo sustentable. El Artículo $2^{\circ}$ determina que la política ambiental nacional deberá cumplir los siguientes objetivos: a) Asegurar la preservación, conservación, recuperación y mejoramiento de la calidad de los recursos ambientales, tanto naturales como culturales, en la realización de las diferentes actividades antrópicas; b) Promover el mejoramiento de la calidad de vida de las generaciones presentes y futuras, en forma prioritaria; c) Fomentar la participación social en los procesos de toma de decisión; d) Promover el uso racional y sustentable de los recursos naturales; e) Mantener el equilibrio y dinámica de los sistemas ecológicos; f) Asegurar la conservación de la diversidad biológica; g) Prevenir los efectos nocivos o peligrosos que las actividades antrópicas generan sobre el ambiente para posibilitar la sustentabilidad ecológica, económica y social del desarrollo; h) Promover cambios en los valores y conductas sociales que posibiliten el desarrollo sustentable, a través de una educación ambiental, tanto en el sistema formal como en el no formal; i) Organizar e integrar la información ambiental y asegurar el libre acceso de la población a la misma; j) Establecer un sistema federal de coordinación interjurisdiccional, para la implementación de políticas ambientales de escala nacional y regional y $\mathbf{k}$ ) Establecer procedimientos y mecanismos adecuados para la minimización de riesgos ambientales, para la prevención y mitigación de emergencias ambientales y para la recomposición de los daños causados por la contaminación ambiental (Congreso de Argentina, 2002).

En el Artículo 4º se hace la interpretación y aplicación de la anterior ley, y de toda otra norma a través de la cual se ejecuta la política Ambiental, están sujetas al cumplimiento de los siguientes principios de: Congruencia: La legislación provincial y municipal referida a lo ambiental debe ser adecuada a los principios y normas fijadas en la presente ley; en caso de que así no fuere, éste prevalecerá sobre toda otra norma que se le oponga, Prevención: Las causas y las fuentes de 
los problemas ambientales se atenderán en forma prioritaria e integrada, tratando de prevenir los efectos negativos que sobre el ambiente se pueden producir, Precautorio: Cuando haya peligro de daño grave o irreversible la ausencia de información o certeza científica no deberá utilizarse como razón para postergar la adopción de medidas eficaces, en función de los costos, para impedir la degradación del medio ambiente, Equidad intergeneracional: Los responsables de la protección ambiental deben velar por el uso y goce apropiado del ambiente por parte de las generaciones presentes y futuras, Progresividad: Los objetivos ambientales deben ser logrados en forma gradual, a través de metas interinas y finales, proyectadas en un cronograma temporal que facilite la adecuación correspondiente a las actividades relacionadas con esos objetivos. Responsabilidad: El generador de efectos degradantes del ambiente, actuales o futuros, es responsable de los costos de las acciones preventivas y correctivas de recomposición, sin perjuicio de la vigencia de los sistemas de responsabilidad ambiental que correspondan. Subsidiariedad: El Estado nacional, a través de las distintas instancias de la administración pública, tiene la obligación de colaborar y, de ser necesario, participar en forma complementaria en el accionar de los particulares en la preservación y protección ambientales. Sustentabilidad: El desarrollo económico y social y el aprovechamiento de los recursos naturales deberán realizarse a través de una gestión apropiada del ambiente, de manera tal, que no comprometa las posibilidades de las generaciones presentes y futuras. Solidaridad: La Nación y los Estados provinciales son responsables de la prevención y mitigación de los efectos ambientales transfronterizos adversos de su propio accionar, así como de la minimización de los riesgos ambientales sobre los sistemas ecológicos compartidos y Cooperación: Los recursos naturales y los sistemas ecológicos compartidos serán utilizados en forma equitativa y racional, El tratamiento y mitigación de las emergencias ambientales de efectos transfronterizos serán desarrollados en forma conjunta (Congreso de Argentina, 2002). 


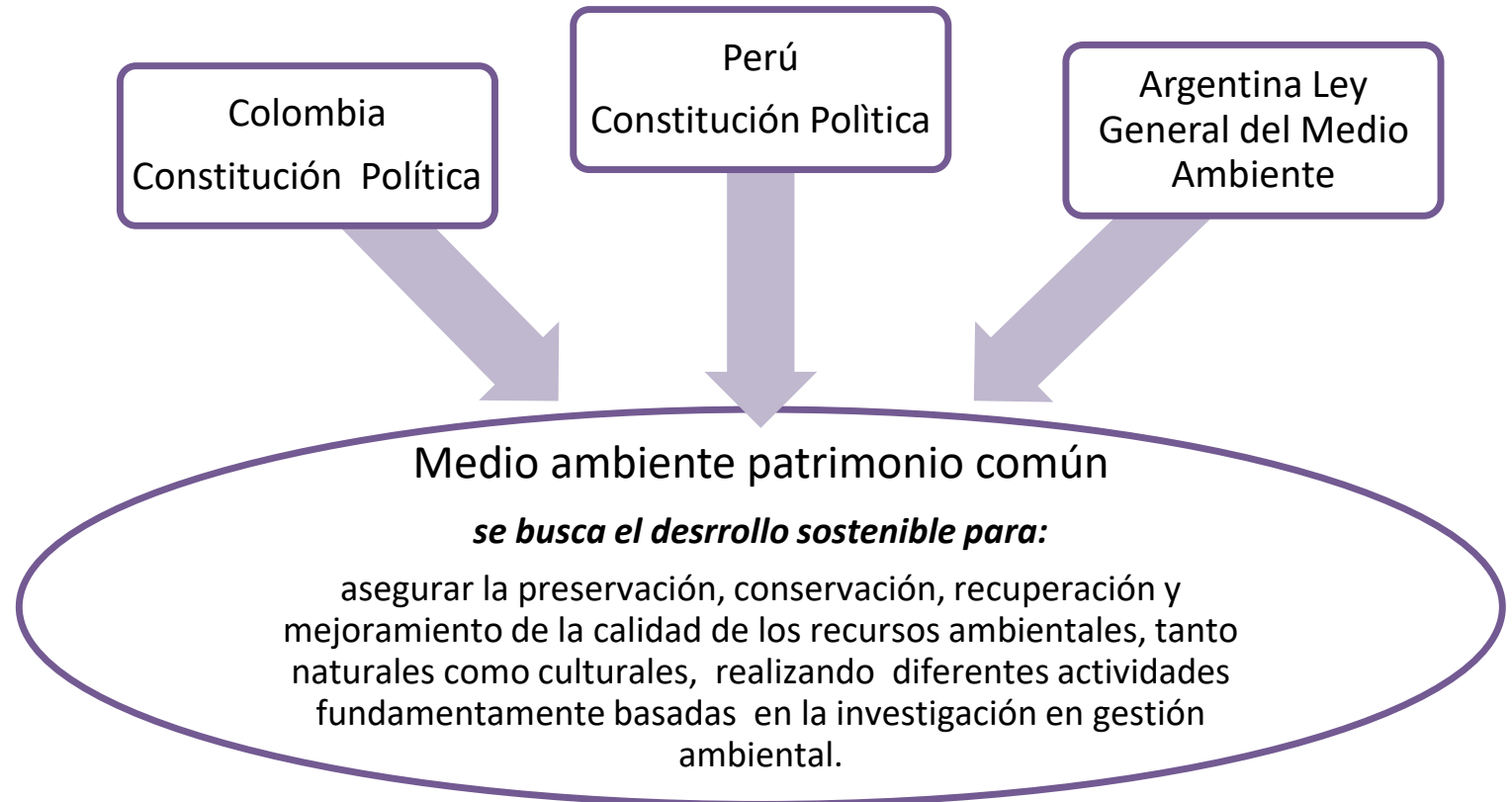

Figura 1. Elementos en común en la normatividad ambiental de estos tres países Latinoamericanos. Adaptado de: EPC 1991, CCDP 2009 y Congreso de Argentina, 2002.

\section{PLANTEAMIENTOS BASADOS EN EL CICLO DE VIDA DE LOS RECURSOS LAS NORMAS EXISTENTES}

El objeto fundamental de la reglamentación ambiental es reducir las presiones en cada etapa del ciclo de vida de los recursos, lo que incluye su extracción o recolección, uso y eliminación final. Se trata por tanto de integrar este concepto de ciclo de vida y de impacto de los recursos en todas las nomas políticas relacionadas con ellos. Este planteamiento se ha ido aplicando sistemáticamente, fundamentalmente en el tratamiento de los residuos. Algunas acciones, como la política integrada de productos o el plan de acción para las tecnologías ambientales, complementan esta integración (UE, 2003).

Por otra parte, las políticas que no están relacionadas con el medio ambiente pero consumen recursos deberán integrar este planteamiento. Ya se han adoptado medidas al respecto, especialmente en el ámbito del transporte y la energía. Además, los estudios de impacto sobre los recursos serán determinantes en 
algunos sectores industriales o de infraestructura. Para reforzar los conocimientos sobre el uso de los recursos y su impacto ambiental, se propone la creación de un centro de datos sobre recursos naturales donde se recopilarán los datos procedentes de varios organismos de análisis e investigación, lo cual Facilitará el intercambio de datos y su puesta a disposición de los responsables políticos. También se requiere elaborar indicadores que permitan controlar y evaluar periódicamente los avances registrados en la consecución del objetivo de la mejora de la eficacia en el uso de los recursos, la disociación entre el uso de los recursos y los impactos ambientales y la disociación entre el crecimiento económico y los impactos negativos para el medio ambiente. Por su parte, se invita a todos los países formar comisiones para elaborar medidas y programas a escala nacional e internacional, especialmente en educación ambiental, formación o incentivos económicos (UE, 2003).

\section{CERTIFICADOS DE INCENTIVO FORESTAL}

En Colombia mediante la Ley 139 de 1994, se creó el certificado de incentivo (CIF), dando cumplimiento por parte del Estado a los artículos 79 y 80 de la Constitución Política, donde éste reconoce a las externalidades positivas de la reforestación tanto los beneficios ambientales y sociales generados son apropiables por el conjunto de la población. Su fin es el de promover la realización de inversiones directas en nuevas plantaciones forestales de carácter protectorproductor en terrenos de aptitud forestal. Podrán acceder a éste las personas naturales o jurídicas de carácter privado, entidades descentralizadas municipales o distritales cuyo objeto sea la prestación de servicios públicos de acueducto y alcantarillado y entidades territoriales, que mediante contrato celebrado para el efecto con las entidades competentes para la administración y manejo de los recursos naturales renovables y del ambiente, se comprometan a cumplir un Plan de Establecimiento y Manejo Forestal (Figura 2), en los términos y condiciones señalados en la presente Ley (Congreso de Colombia ,1994).

El certificado de incentivo forestal es un documento otorgado por la entidad competente para el manejo y administración de los recursos naturales renovables 
y del medio ambiente, que da derecho a la persona beneficiaría a obtener directamente al momento de su presentación, por una sola vez y en las fechas, términos y condiciones que específicamente se determinen, las sumas de dinero que se fijen conforme a lo establecido por la ley, por parte de la entidad bancaria que haya sido autorizada para, el certificado es personal y no negociable. Consiste en una bonificación en efectivo de los costos de siembra de plantaciones forestales con fines protectores-productores en terrenos de aptitud forestal, del $50 \%$ para especies introducidas y nativas. Por los costos totales netos de manejo del segundo hasta el quinto año se reconoce hasta un $50 \%$ para ambos casos (Congreso de Colombia, 1994 y 2009).

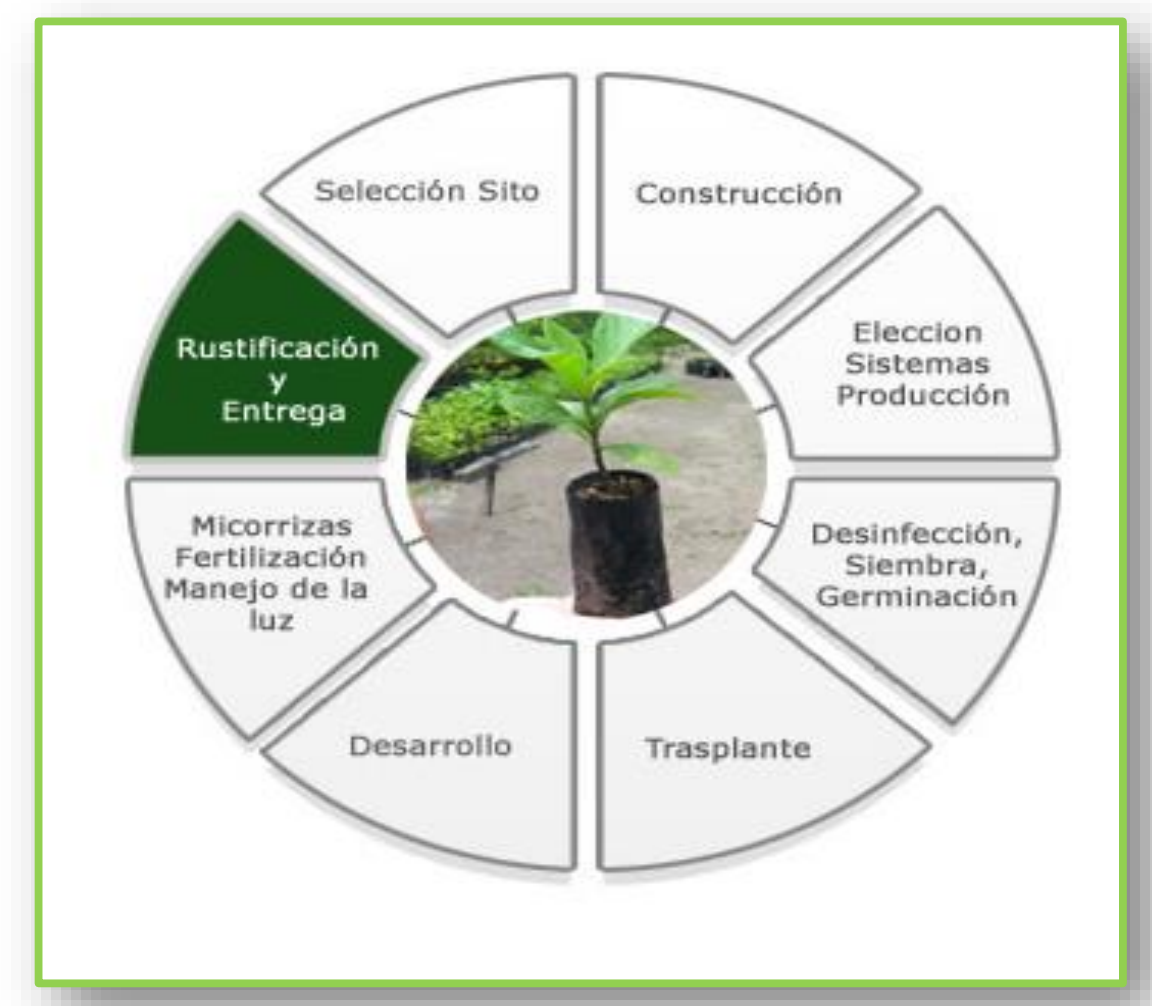

Figura 2. Procedimientos para el establecimiento y trasplante de especies forestales, tomado de El semillero, (2012).

En el Artículo 16 de la ley 139 se creó el Comité Asesor de Política Forestal (CAPF), con el fin de coordinar la ejecución de las políticas relacionadas con el subsector forestal, conformado por el Ministro del Medio Ambiente o su delegado, 
quien lo presidirá, el Ministro de Agricultura o su delegado, el Gerente General del Instituto Colombiano Agropecuario, ICA, o su delegado, el Jefe de la Unidad de Desarrollo Agrario del Departamento Nacional de Planeación, un representante de las Corporaciones Autónomas Regionales, el Presidente de la Asociación Colombiana de Reforestadores ACOFORE, el Presidente de la Corporación Nacional de Investigación y Fomento Forestal, el Director del Instituto de Ciencias Naturales de la Universidad Nacional, el Presidente de la Asociación Colombiana de Estudios Vegetales "in vitro", un representante de las Organizaciones no Gubernamentales de Carácter Ambiental y un representante de la Asociación de Secretarios de Agricultura. La evaluación, verificación de campo, seguimiento y control del Plan de Establecimiento y Manejo Forestal, corresponderá a la respectiva entidad competente para la administración y manejo de los recursos naturales renovables y del medio ambiente, la cual podrá delegar total o parcialmente tales funciones en otras entidades públicas o privadas (Congreso de Colombia, 1994).

\section{LICENCIA AMBIENTAL}

El Ministerio del Medio Ambiente (MMA), mediante decreto 1753 de 1994, dispuso las normas para las licencias ambientales para Colombia, en su Artículo 2. Conceptúa que La Licencia Ambiental (LA) es la autorización que otorga la autoridad competente, mediante acto administrativo, a una persona, para la ejecución de un proyecto, obra o actividad que conforme a la ley y a los reglamentos, puede producir deterioro grave a los recursos naturales renovables 0 al medio ambiente $o$ introducir modificaciones considerables o notorias al paisaje, y en la que se establecen los requisitos, obligaciones y condiciones que el beneficiario de la LA, debe cumplir para prevenir, mitigar, corregir, compensar y manejar los efectos ambientales del proyecto, obra o actividad autorizada.

En el Artículo 3. del mismo decreto se especifica que elementos debe tener una licencia ambiental (Figura 3), Cuando el beneficiario de una LA deba prestar una póliza de cumplimiento o una garantía bancaria, a favor de la autoridad ambiental competente, según ésta lo determine, teniendo en cuenta los riesgos inherentes 
del proyecto, obra, actividad y otras garantías ya constituidas, con el fin de asegurar el cumplimiento de los términos, requisitos, condiciones, exigencias $u$ obligaciones de la LA, tales garantías serán prestadas hasta por un monto máximo del 30\% del valor anual del plan de manejo. La póliza deberá ser renovada anualmente y tener vigencia durante la vida útil del proyecto y hasta por dos años más a juicio de la autoridad ambiental. Los recursos provenientes de la ejecución de la póliza de cumplimiento o de la garantía bancaria se destinarán a una subcuenta del Fondo Nacional Ambiental, con el objeto de utilizarla en la compensación, corrección, mitigación y manejo de los impactos y efectos causados (MMA, 1994). Según el decreto 1753 en Colombia existen tres modalidades de LA que son:

1-Licencia Ambiental Ordinaria: Es la cual se establecen los requisitos, condiciones y obligaciones que el beneficiario de la LA debe cumplir para prevenir, mitigar, corregir, compensar y manejar los efectos ambientales del proyecto, obra o actividad autorizada, sin disponer sobre el otorgamiento de los permisos, autorizaciones o concesiones para el uso, aprovechamiento o movilización de los recursos naturales renovables (MMA, 1994).

2. Licencia Ambiental Única: Es la otorgada por la autoridad ambiental competente y que, a solicitud del peticionario, incluye los permisos, autorizaciones o concesiones, necesarios para el desarrollo del proyecto, obra o actividad. La vigencia de estos permisos, concesiones y autorizaciones, de acuerdo con su naturaleza, podrá ser la misma de la LA. Para su otorgamiento se observarán las siguientes reglas: a) La autoridad ambiental competente ante la cual la solicita, asumirá la competencia para el otorgamiento de los permisos, autorizaciones y concesiones a que haya lugar; para ello observará las normas que en cada región sean aplicables; b) El otorgamiento de los permisos, autorizaciones y concesiones solicitados se hará en el mismo acto de otorgamiento de la LA; c) La autoridad ambiental solicitará a las entidades cuya competencia asume en virtud de la solicitud de la LA Única, la información técnica, jurídica y administrativa que sea indispensable para decidir sobre el otorgamiento de los permisos, autorizaciones y 
concesiones necesarias para el desarrollo del proyecto, obra o actividad; d) El otorgamiento de los permisos, autorizaciones y concesiones se comunicará formalmente a la entidad respectiva cuya competencia en cada caso se asume (MMA, 1994).

3-Licencia Ambiental Global: puede ser ordinaria o única. Es de competencia exclusiva del Ministerio del Medio Ambiente y en virtud de ella se autorizan todas las obras o actividades relacionadas con la explotación de campos petroleros y de gas. Cuando la Licencia Ambiental Global sea Ordinaria, el otorgamiento de ésta no releva al beneficiario de la obligación legal o reglamentaria de obtener los permisos, autorizaciones o concesiones que sean necesarios dentro del campo de producción autorizado, ni del cumplimiento de sus condiciones y obligaciones específicas. Para el desarrollo de cada una de las obras o actividades definidas en la etapa de explotación será necesario presentar un plan de manejo ambiental conforme a los términos, condiciones y obligaciones establecidas en la Licencia Ambiental Global Ordinaria (MMA, 1994).

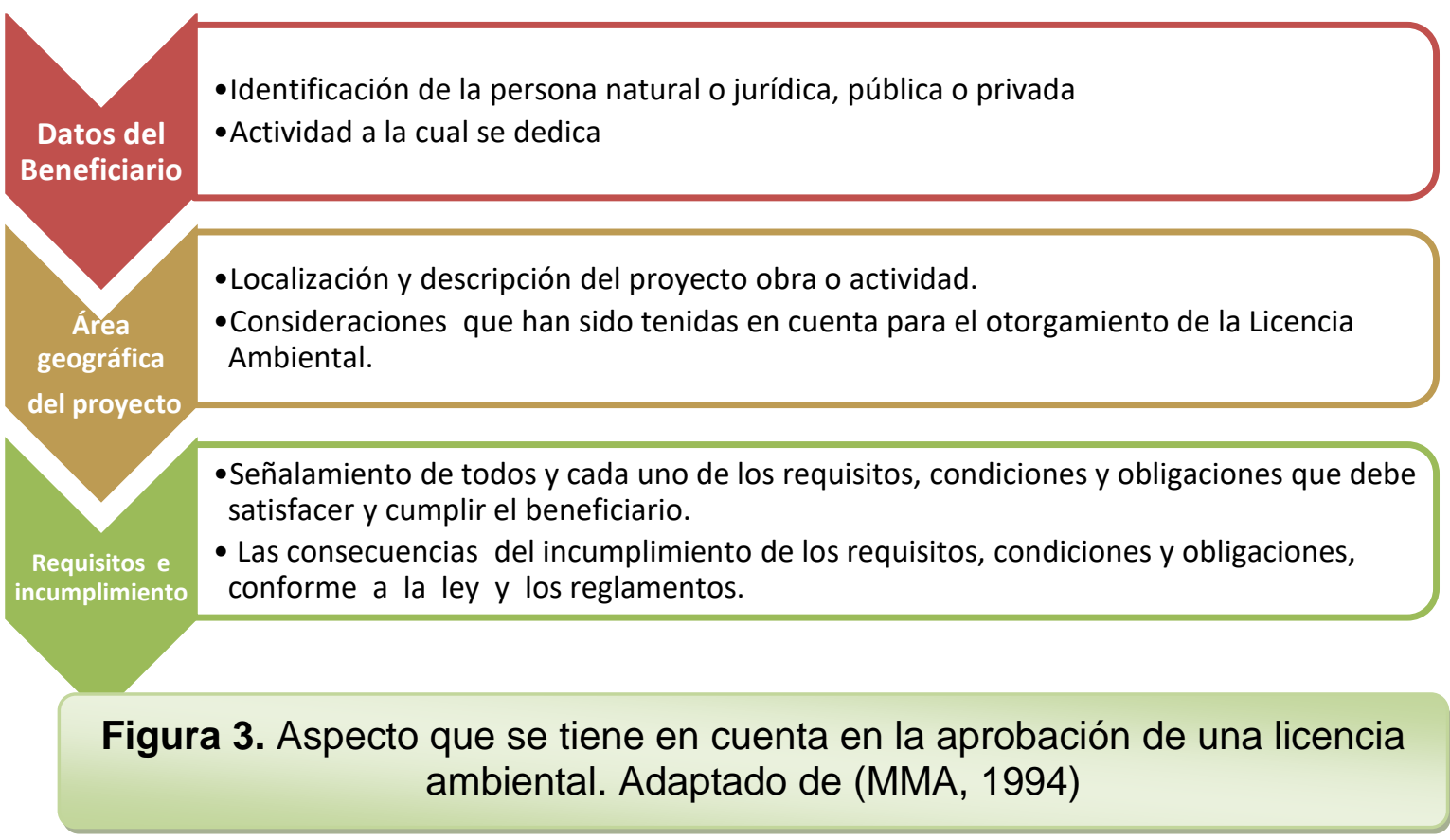

La obtención de la LA Ordinaria y Global Ordinaria, es requisito previo para el otorgamiento de los permisos, autorizaciones y concesiones que se requieran 
conforme a la ley o los reglamentos. La obtención de la LA es condición previa para el ejercicio de los derechos que surjan de los permisos, autorizaciones, concesiones y licencias que no sean de competencia de la autoridad ambiental. El término de la Licencia Ambiental será el mismo de la duración del proyecto, obra o actividad. Sin embargo, la autoridad ambiental, de oficio o a petición de parte, podrá establecer un término diferente teniendo en cuenta el Estudio de Impacto Ambiental o la naturaleza del proyecto, obra o actividad, (MMA 1994).

\section{ACTIVIDADES CON ESPECIES AGROFORESTALES CON FINES ECONÓMICOS}

La ley 1377 del 2010 del Congreso de Colombia tiene por objeto definir y reglamentar las plantaciones forestales y sistemas agroforestales con fines comerciales. En el Artículo 2. define: 1. La actividad forestal con fines comerciales es el cultivo de especies arbóreas de cualquier tamaño originado por la intervención directa del hombre con fines comerciales o industriales y que está en condiciones de producir madera, productos forestales no maderables $y$ subproductos, 2. El Sistema agroforestal es la combinación en tiempo y espacio de plantaciones forestales con fines comerciales asociadas con cultivos agrícolas o actividades pecuarias. 3. Vuelo forestal: Es el volumen aprovechable sobre el cual el titular o el propietario de un cultivo forestal con fines comerciales tiene derecho para constituir una garantía. Para todos los efectos jurídicos, se entiende que los árboles son bienes muebles. 4. Certificado de Incentivo Forestal, CFI, para apoyo de programas de reforestación comercial: Es el documento que otorga a su titular el derecho a obtener directamente, al momento de su presentación, los apoyos o incentivos económicos que otorga el Gobierno Nacional para promover las actividades forestales y sistemas agroforestales con fines comerciales. 5 . Remisión de movilización: Es el documento en el que se registra la movilización de madera 0 de productos forestales de transformación primaria provenientes de actividades forestales 0 sistemas agroforestales con fines comerciales debidamente registrados (Congreso de Colombia, 2010). 
El Ministerio de Agricultura y Desarrollo Rural, como organismo rector de la producción agropecuaria, forestal y pesquera, es la única entidad competente para formular las políticas del sector forestal comercial y sistemas agroforestales, así como el otorgamiento y reglamentación del Certificado de Incentivo Forestal, CIF, para apoyo de programas de reforestación comercial. Además asumirá las funciones atribuidas en la Ley 139 de 1994 a las entidades competentes para la administración y manejo de los recursos naturales renovables en relación con el Certificado de Incentivo Forestal, CIF, para apoyo de programas de reforestación comercial. El Ministerio de Agricultura y Desarrollo Rural determinará anualmente la distribución del presupuesto asignado para este incentivo (Congreso de Colombia 2010).

En el Artículo 6을 el Ministerio de Agricultura y Desarrollo Rural, establece la implementación de un Sistema de Control de conformidad con lo consagrado en esta ley y las normas existentes sobre el tema, el cual permitirá garantizar el debido aprovechamiento de los productos de las plantaciones comerciales forestales y de los sistemas agroforestales. Este Sistema de Control, estable mecanismos de identificación de los productos provenientes de las plantaciones forestales $y$ de los sistemas agroforestales con fines comerciales, e incluye métodos de control desde el registro de las personas naturales o jurídicas que se dediquen a esta actividad, su aprovechamiento, movilización y comercialización, el cual deberá ser adoptado por los titulares de los registros. Así mismo, implementará un medio de información actualizado permanente, que contenga aspectos tales como número de registro del cultivo o sistema agroforestal, ubicación, áreas y especies registradas, sembradas y aprovechadas, nombre e identificación del propietario o tenedor del predio y de la plantación, volúmenes y descripción de los productos, origen, ruta y destinos de comercialización, modo de transporte e identificación del vehículo y del transportador, entre otros. Estos sistemas de control e información, se desarrollarán bajo el principio de transparencia y autorregulación, por lo cual esta información será pública y de fácil acceso (Congreso de Colombia, 2010). 


\section{PROTECCIÓN DE BOSQUES NATURALES Y ECOSISTEMAS ESTRATÉGICOS}

No podrán establecerse cultivos forestales o sistemas agroforestales con fines comerciales en bosques naturales, áreas forestales protectoras, áreas de manejo especial o cualquier otra categoría de manejo, conservación o protección que excluya dicha actividad, así como ecosistemas estratégicos, tales como páramos, manglares, humedales. Los caminos necesarios para adelantar el aprovechamiento forestal dentro de los cultivos forestales y sistemas agroforestales con fines comerciales son parte integrante de estos y no estarán sometidos a permisos o requisitos adicionales distintos a los previstos en la presente ley. Cuando en la construcción de una carretera se requiera del uso o afectación de recursos naturales renovables, se deberán tramitar y obtener ante las autoridades ambientales competentes las autorizaciones o permisos correspondientes. Para efectos de planificar las actividades de reforestación comercial, el Ministerio de Agricultura y Desarrollo Rural o la entidad que esta delegue, informará cuáles son las zonas potenciales para adelantar el desarrollo de estos cultivos. Lo anterior, sin perjuicio de las competencias constitucionales y legales que sobre la materia tienen los Concejos Municipales. Para los efectos de las actividades forestales y sistemas agroforestales con fines comerciales el instituto Colombiano Agropecuario, ICA, es la entidad competente para regular las actividades de control, prevención y erradicación de plagas y enfermedades (Figura 4) (Congreso de Colombia, 2010).

\section{CONCLUSIÓN}

Según los planteamientos filosóficos, la ecología y el medio ambiente, como cualquier ciencia se fundamenta en la obtención de datos que permitan confrontar las diferentes descripciones antes mencionadas. Estas descripciones 0 explicaciones de los fenómenos que ocurren en la naturaleza se denominan hipótesis, elemento central sobre el que se articula el método científico. Aunque existen varias metodologías el biomonitoreo sigue siendo una guía efectiva, para estimar los efectos negativos de cualquier factor en distintos organismos, y además son bioindicadores para determinar la toxicidad de compuesto químicos. 


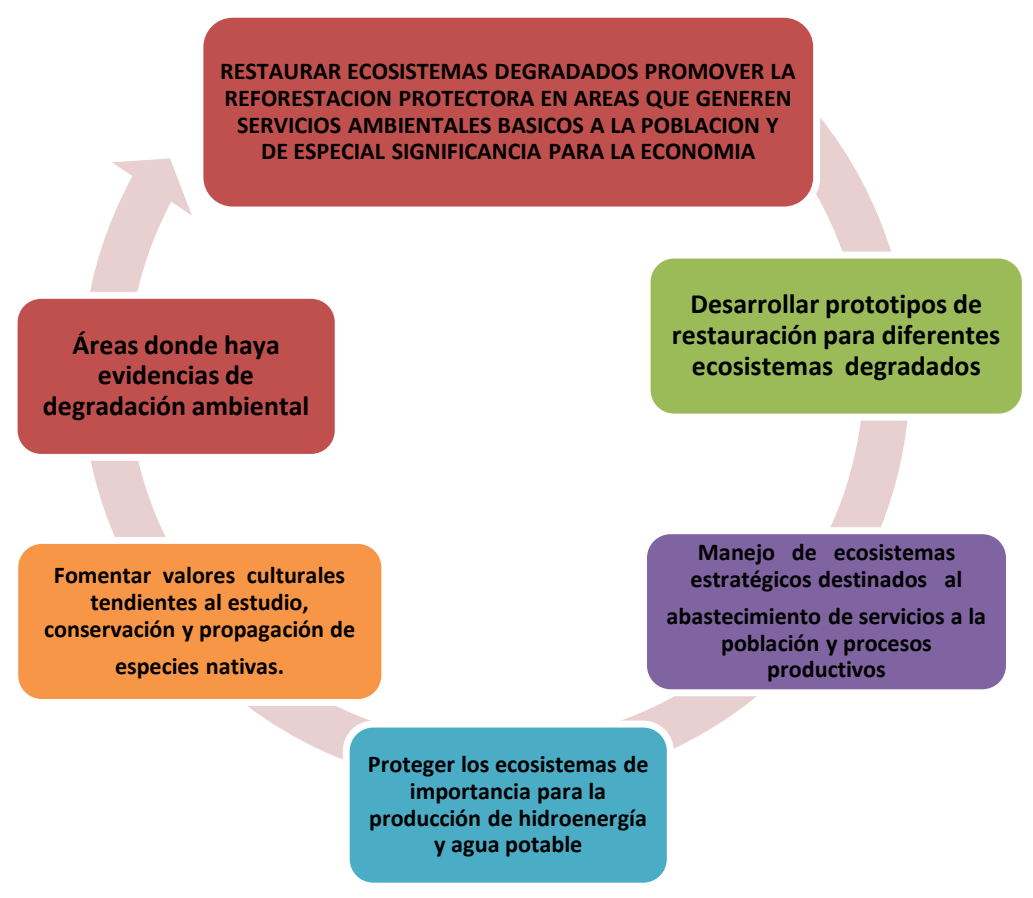

Figura 4. Plan estratégico para la restauración ecológica Adaptado de MMA 1998.

Para que los resultados sean más confiables, las metodologías de investigación para los ecosistemas y medio ambiente requieren de un mayor número de unidades experimentales para probar de manera adecuada otros modelos que puedan ser manejados para las matrices de evaluación ecológica y del medio ambiente.

Se comprueba que las especies tienen comportamientos diferentes, como el caso de la abeja Apis mellifera, se adaptó perfectamente a un ambiente altamente industrializado, sin que se observaran modificaciones significativas de su conducta ni alteraciones en su biología. Se logró con éxito el desarrollo, construcción y adaptación de los diferentes elementos técnicos necesarios para la realización de su monitoreo ambiental, mediante el uso de Estaciones de Biomonitoreo Gemelas con Análisis Simultáneos, con una colmena especialmente adaptada en su forma y tamaño, un muestreador de material vivo, un contador de abejas y un protocolo de observación para monitoreo ambiental. 


\section{REFERENCIAS BIBLIOGRÁFICAS}

1. CEPAL "Equidad, Desarrollo y Ciudadanía", preparado por CEPAL para el Vigesimoctavo Período de Sesiones celebrado en México, D.F., en abril de 2000.

2. Congreso de Argentina. El Senado y Cámara de Diputados de la Nación Argentina reunidos en Congreso, sancionan con fuerza de Ley: Ley general del ambiente. Ley Nacional 25.675. Bien jurídicamente protegido. Sancionada: 6 de noviembre de 2002.

3. Congreso de Colombia, Ley 139 DE 1994 Por la cual se crea el certificado de incentivo forestal y se dictan otras disposiciones Diario Oficial No. 41.401., Bogotá, Colombia de 22 de junio de 1994.

4. Congreso de Colombia Ley 1328 por la cual se dictan normas en materia financiera, de seguros, del mercado de valores y otras disposiciones.15-072009.

5. Congreso de Colombia- Ley 1377. Por medio de la cual reglamenta la actividad de reforestación comercial. Diario Oficial No. 47.586 de 8 de enero de 2010.

6. El Congreso Constituyente Democrático de Perú (CCDP). Constitución política 2009. Recuperado 23 de abril 2012. Disponible En: http://www.allpa.org.pe/content/constitucion-politica-del-peru

7. EPA. Evaluación y manejo de riesgos: sistema para la toma de decisiones. EPA. Traducción de OPS, 1996.

8. El Pueblo de Colombia (EPC). Constitución Política de Colombia. 1991. Recuperado 23 de abril 2012. Disponible En: http://www.banrep.gov.co/regimen/resoluciones/cp91.pdf

9. El Congreso Constituyente Democrático de Perú (ECCDP). Constitución política 2009. Recuperado 23 de abril 2012. Disponible En: http://www.allpa.org.pe/content/constitucion-politica-del-peru

10. El semillero. Producción de vivero. 2012. Recuperado 28 de abril 2012. Disponible http://elsemillero.net/nuevo/index.php?option=com wrapper\&view=wrapper\&lt emid=360

11. Ministerio del Medio Ambiente (MMA). Licencias ambientales decreto 1753 de Agosto 3 de 1994.

12. Ministerio del Medio Ambiente (MMA). Plan estratégico para la restauración ecológica y el establecimiento de bosques en Colombia. Plan verde. 1998. Recuperado 28 de abril 2012. Disponible En: http://www.finagro.com.co/html/cache/HTML/SIS/Reforestacion/pverde[1].pdf

13. Palma A. Biomonitoreo. Recuperado 21 de abril 2012. Disponible En: http://www2.udec.cl/ /palma/documentos/biomonitoreo.html

14. Porrini, C. La abeja como bioindicador del medio ambiente. Vida Apícola, 23 de Febrero de 2006.

15. Segretin M.E .Biomonitoreo ambiental y tratamiento de efluentes. CONICET FECyN/UBA. Recuperado 21 de abril 2012. Disponible En: 
http://www.argenbio.org/adc/uploads/pdf/Biomonitoreo\%20y\%20tratamiento\%2 0de\%20efluentes.pdf

16. Szczupak M. ¿Qué es el biomonitoreo?. 2010. Recuperado 21 de abril 2012. Disponible En: http://lular.es/a/entorno/2010/09/Que-es-el-biomonitoreo.html 2010.

17. Unión Europea (UE). Hacia una estrategia temática para el uso sostenible de los recursos naturales. 2003. Recuperado 23 de abril 2012. Disponible En: http://europa.eu/legislation summaries/environment/sustainable development/ |28167 es.html

18. Vizcaino S. Introducción al derecho del medio ambiente Madrid, 1996. 